\title{
THE POSITIONING ERRORS GENERATED BY XSENS MVN INDUCTIVE SYSTEM DURING THE ANALYSIS OF „PUSH-UPS” EXERCISE, USING THE „SINGLE LEVEL” SCENARIO, BEFORE AND AFTER THE CALLING OF "REPROCESS" FUNCTION
}

\author{
Badea Cristian Radu ${ }^{1}$, Badea Sorin Ionut ${ }^{2}$ \\ ${ }^{1,2}$ National Institute of Research and Development in Mechatronics and Measurement Technique, Bucharest \\ E-mail: adresacontact@gmail.com,sorin ib@yahoo.com
}

\begin{abstract}
The study aimed to perform motion analysis using Xsens's MVN inertial system, in order to assess the positioning errors generated by this system, during the execution of „Push-ups”-type exercise on the rigid floor, both before and after the calling of "REPROCESS" function.

This study it's not meant to decide the quality of Xsens MVN system, representing just a beginner's findings on his first contact with any analysis sistem.

The analysis was of a qualitative nature, concentrating on the positioning precision with respect to the global reference system, the imposed exercise being performed freely without the help of any devices, but with regard to the following:

- each of „Push-ups”-type individual exercise was performed with the human subject positioned in the same place, imposing it to maintain the same distance between the feet / hands;

- the data obtained before and after the use of „REPROCESS” function, were compared with each other, in order to verify the system's ability to correct the positioning errors;

- the chosen scenario for the analysis of this exercise was „Single Level”.

The results of the analysis were:

a) The system presents both global positioning errors and local errors, generated by the MEMS located on the feet;

b) The contact points are being transferred from the palms to the toes and vice versa, throughout the entire session.

c) After the calling of "REPROCESS" function, the errors decrease but they are not completely eliminated, being reduced from the $\mathrm{cm}$ to the $\mathrm{mm}$ order.
\end{abstract}

Keywords: Positioning errors, Inductive motion analysis system, Mechatronic, MEMS, "Push-ups"Type exercise, "REPROCESS" Function.

\section{Introduction}

A modern mechatronic inertial motion analysis system is based on a MEMS sensory network, each of which contains a combination of inertial and magnetic sensors, whose signals are processed by means of advanced processing algorithms and transferred to biomechanical models.

The role of MEMS within a mechatronic motion analysis systems is to provide accurate data on body's segments orientation, this being achived by processing data from the sensors contained within each MEMS, with the aid of a microcontroller (also contained within each MEMS). The resulting data are providing information about acceleration, angular velocity, magnetic field value and in some configurations even pressure.
In general the information provided by MEMSbased mechatronic inertial motion analysis systems are:

- joint angles;

- kinematics of body segments;

- the global position of body segments;

- body's center of mass;

- data from sensors.

Regarding real-time analysis capability, the latency of such systems is around $30 \mathrm{~ms}$.

The range for wireless systems is approx. 20$50 \mathrm{~m}$, and the update rate of MEMS is approx. $1000 \mathrm{~Hz}$, the transmission being performed at a frequency between $60-240 \mathrm{~Hz}$.

Lately, specialized terminology has undergone some changes in terms of sampling frequency, which has been replaced by the "update rate". This was due to the fact that the MEMS signal is sampled at very high frequencies $(1000 \mathrm{~Hz})$ in order to maintain high 
accuracy, but at this frequency, the data can not be transmitted wirelessly (frequent in this case being around of $100 \mathrm{~Hz}$ ). Therefore, with the help of specific algorithms, data from MEMS-ri are transmitted in incremental form, and if a wireless communication interruption occurs, the data is temporarily stored and sent in full when the connection is restored.

Modern, MEMS based mechatronic inertial motion analysis systems are considered to be immune to magnetic distortion.

In the field of inertial motion analysis systems, Xsens MVN is considered to be one of the most performant system. An extremely important feature, provided by this system, which supposedly helps to eliminate errors, is the "remapping" of the magnetic field, this action being performed with the aid of a specialized software, named Magnetic Field Mapper, which allows the placing of MEMS on ferromagnetic surfaces (such as the case of MEMS located on the prosthetic areas), as well as performing motion analyzes in areas with disturbing magnetic fields.

In the past decade, inertial motion capture systems have seen a growing use of their applications, including their use in animation applications, games, movies and virtual reality as well as in motion analysis applications for medicine, ergonomics and sports.

Compared to alternative motion analysis systems, such as optical or mechanical, that are based on external transmitters and / or video camera networks, restricting their usage to special laboratory conditions and / or large workspace, the inertial systems, doesn't require an external infrastructure, which recommends them for applications with a high level of portability and a variety of usage conditions: enclosed or open spaces, small and / or large areas.

Despite this tremendous advantage over alternative motion analysis systems, they present a major disadvantage, namely the positional drift (positional error). The estimation of the positions and orientation, of the body segments of the human subject under analysis, is performed by means of a sensor to segment calibration procedure, starting from the information on position and orientation, received from the MEMS, which then are transfer to a biomechanical (scaled) model of the human body. This calibration introduces errors that may affect performance.

\section{Xsens's MVN Inertial Analysis System Specifications}

Starting from the above mentioned, the main objective of this study is to explore the possibilities of analysis provided by the inertial motion analysis system MVN, developed by Xsens and to determine its limitations:

The most important specifications of the Xsens's MVN inertial motion analysis system are:

a) Full immunity to disturbing electromagnetic field during live sessions:

- eliminating the orientation error caused by magnetic interference with ferromagnetic materials;

- limiting the working environment restrictions, where the system can be used, without problems, inside the enclosures with heavily armed wall, or in the proximity of metal objects, in the cars, airplanes, or trains.

b) High resolution reprocessing engine. This allows off-line reprocessing of information, which leads to an increase in the quality of the data provided by the system. The basic features of this reprocessing engine are:

- correcting the "slipping" of the foot's position;

- corrections of the multi-point type contact;

- Improving the data consistency.

- Improving the precision of 3D articulation and kinematics data;

- Improving the precision of the global 3D positioning estimation;

c) Live detection of multiple contact points, enabling analyzes such as:

- walking in the hands;

- climbing;

- stairs climbing;

- gymnastics.

d) The remote-control application, which has the following facilities:

- Synchronizing with your mobile phone camera

- remote control of the system's basic functions;

e) Global 3D positioning error in a live analysis session is approximately $1 \%$ of the distance traveled;

f) Multi-level capture within live analysis sessionsthe system allows the user to perform measurements of jumps and running, in that the system can detect height changes in a global 3D coordinate system;

g) The internal sampling rate of MEMS, which captures movements, is $1000 \mathrm{~Hz}$;

h) Modular articulations based on human anatomical structures allowing 6 degrees of freedom;

i) Lack of the orientation constraints applied to the segments, as well as the angular constraints, applied to the joints (the virtual joints behave in exactly the same manner as the human ones). The information obtained is not manipulated by the system to create the appearance of natural movements, but 
accurately reflects the values measured by the MEMS of the system.

\section{Xsens's MVN Inertial Motion Analysis System Architecture}

Like the vast majority of inertial motion analysis systems, the Xsens MVN system consists of a hardware subassembly and a software subassembly.

\subsection{Hardware subassembly}

The hardware subassembly used in the study is called MVN Link and is a 3D kinematics measurement system adapted to the human body, composed of a network of MEMS, interconnected by means of electric cables, mounted / mountable, in predetermined positions, on a elastic "Lycra"-type suit, the latter allowing the user to have a maximum freedom of movement, but also to reduce the time required for the positioning / repositioning of all the MEMS. MVN Link can be used both indoors and outdoors, on rough terrain, in low-light areas. The results provided by MVN Link do not require postprocessing, as the MEMS used do not suffer from the occlusion phenomenon, as with optical markers. The data provided by this system can also be easily used by other software applications.

Features and basics of MVN Link:

a) Contains 17 MEMS for motion capture;

b) System Output Frequency: 240Hz;

c) Internal signal sampling frequency: $1000 \mathrm{~Hz}$;

d) Battery life between two loads: 10 hours;

e) Interconnecting cables have KEVLAR reinforced shield to withstand the wear;

f) MEMS's specifications:

- static Accuracy (Roll/Pitch) 0.5 deg RMS;

- static Accuracy (Heading/Yaw) 1 deg RMS;

- dynamic Accuracy (Roll/Pitch) 0.75 deg RMS;

- dynamic Accuracy (Heading/Yaw) 1.5 deg RMS;

- accelerometer range: $+/-160 \mathrm{~m} / \mathrm{s} 2$;

- gyroscope range: + /- $2000 \mathrm{deg} / \mathrm{s}$;

g) the possibility of direct connection, through the dedicated cable (ETHERNET to USB), of the central sensory unit (Body Pack) to the computer;

h) action range of approximately $150 \mathrm{~m}$ when using the WiFi Access Point router;

i) the possibility of extending the range by adding additional WIFI access points;

j) the possibility of switching / changing the WIFI bandwidth between 2.4 and $5 \mathrm{GHz}$, to increase communication stability;

k) the possibility of carrying out independent capture sessions of a calculation system of up to 10 hours;

i) the ability to retransmit data packets, due to the buffer that can store information for max. $10 \mathrm{~min}$; j) possibility of synchronization with external devices, via two internal and two external ports, type BNC;

k) possibility of synchronization with other data acquisition systems, by TTL pulses type $0-3,3 \mathrm{~V}$;

l) possibility of adding up to 4 MEMS for application on props used in the analysis;

m) the possibility of giving up the "Lycra" suit and the use of alternative systems for mounting / positioning MEMS;

\subsection{Software subassembly}

The software subassembly used in the study is called MVN Analyze.

Features and basics of MVN Analyze:

a) The software allows the movements to be visualized through the aid of a virtual dummy that contains 22 joints and 23 segments;

b) Provides information on:

- Global 3D positioning;

- 3D speed coordinates;

- 3D acceleration coordinates;

- 3D angles coordinates;

c) allows visualization of $3 \mathrm{~d}$ coordinates of the analysed human's center of mass;

d) allows real time display of speed, position, orientation, joint angles, center of mass, acceleration;

e) the possibility of exporting / displaying the contact points;

f) Exporting files usable by LabView, Excel and software capable of viewing C3D files.

\section{The objective of the study}

The study aimed to perform motion analysis using Xsens's MVN inertial system, in order to assess the positioning errors generated by this system. The analysis was of a qualitative nature, concentrating on the positioning precision with respect to the global reference system, the imposed exercise being performed freely without the help of any devices.

This study it's not meant to decide the quality of Xsens MVN system, representing just a beginner's findings on his first contact with any analysis sistem.

The chosen exercise for this analysis was „Pushups".

In order to properly evaluate the positioning errors of the Xsens MVN motion analysis inductive system, the following measures were taken:

a) For the analysis, a healthy and emotionally balanced, male subject was chosen, with overaverage motor skills;

b) Each of „Push-ups”-type individual exercise, within each analysis session, was performed with the human subject positioned in the same place, imposing it to maintain the same distance between the feet / hands; 
c) The "REPROCESS" function was used, so that the data obtained before and after its use were compared with each other, in order to verify the system's ability to correct the positioning errors.

d) In order for the proposed goal to get achieved, out of the four scenarios provided by the MVN Analyze software, the chosen scenario for the analysis of this exercise was „Single Level”.

\section{The Chosen Analysis Session and its Conclusions.}

The analysis has been focused on the following exercise: execution of „push-ups”: on rigid floor.

In total, 5 analysis sessions were performed for this exercise. Of these, the most representative 3 sessions were then chosen and in the last phase, after a thorough analysis, only one session was selected, considered to be representative this exercise.

A set of 5 sessions per day was performed, with each exercise, inserting a day break after each measurement day, so that fatigue does not affect the results of the analysis. In the analysis, the duration of each session was determined in relation to the level of phisical stress (the degree of fatigue) imposed by the exercise, this being only an indicative factor, the sessions consisting of a predetermined number of repetitions.

In each chosen session, only that set of consecutive frames considered to be representative for the exercise was used, i.e. the beginning and the end set of frames were removed, because in those frames, the human subject under analysis was preparing for the start of the exercise, or was completed the exercise, and/or because of the physical stress involved, he was getting out of balance.

The human subject, is digitally represented by a virtual dummy, the reference plane of the analyzed motion is digitally represented by a virtual reference plane, having the aspect of a grid and the position of the right heel, while he was in the "N" pose, as required by the calibrating process, it is represented by a red triangle.

The analysis of the images was done by relating the positions of the virtual dummy's body segments to the lines and columns that make up the grid from the virtual reference plane, to the position of virtual reference marker, of the calibration's origin and to the global coordinate system (see Fig.1).

The intersection between the baseline of the virtual reference marker, of the calibration's origin and the perpendicular on this line, it's considered the origin of the analysis.

The first grid line, on the virtual reference plane, which contains the virtual reference marker, is considered the upper line number one, and the one under the virtual reference marker is considered the bottom line number one, in the analysis. The upper lines are in the positive direction of the $0 \mathrm{X}$ axis, and the lower lines in the negative direction of the same axis. The first column containing the right half of the virtual reference marker is considered the right column number one and the first column containing the left half of the virtual reference marker, it's considered the left column number one. The columns situated to the left of the virtual reference marker are in the positive direction of the axis $0 Y$ and the ones on the right in the negative direction of this axis. In the Y0Z plane the points above the virtual reference plan are considered positive and the ones below are considered negative.

In the figures below, the blue dots represent the points of contact that materialize the contact between human subject and those elements, in the work space, required to achieve the imposed movement (eg contact of the palms and toes of the feet with the ground during „Push-ups”).

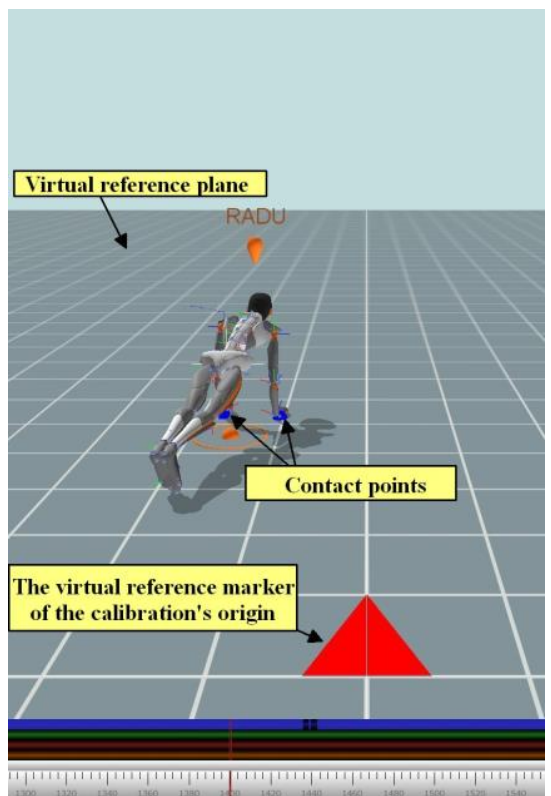

Figure 1: The reference elements for the analysis

Each figure containes two views:

- view a) - obtained without post-processing of the measurement data (without using the "REPROCESS" function provided by the Xsens MVN software);

- view b) - obtained after post-processing of the measurement data, using the "REPROCESS" function provided by the Xsens MVN software.

In the following, we will present the exercise under analysis, the corresponding scenarios, the number of useful repetitions, the number of useful frames, the elements of interest for the analysis and the final results:

- Name of the exercise: Push-ups;

- Software Scenario: Single Level;

- Total number of useful repetitions: 21; 
- Number of useful frames: 5400;

- The segmewnts with contact points: the hands and toes;

- Elements of interest:

- maintaining the position in relation to the global positioning system / virtual reference marker's position, as well as with the virtual reference plan;
- the existence of contact points with the floor at the level of the toes and of the hands;

In this analysis the virtual reference plan is located at the ground level, and the contact points will be found at the level of the hands and of the toes.
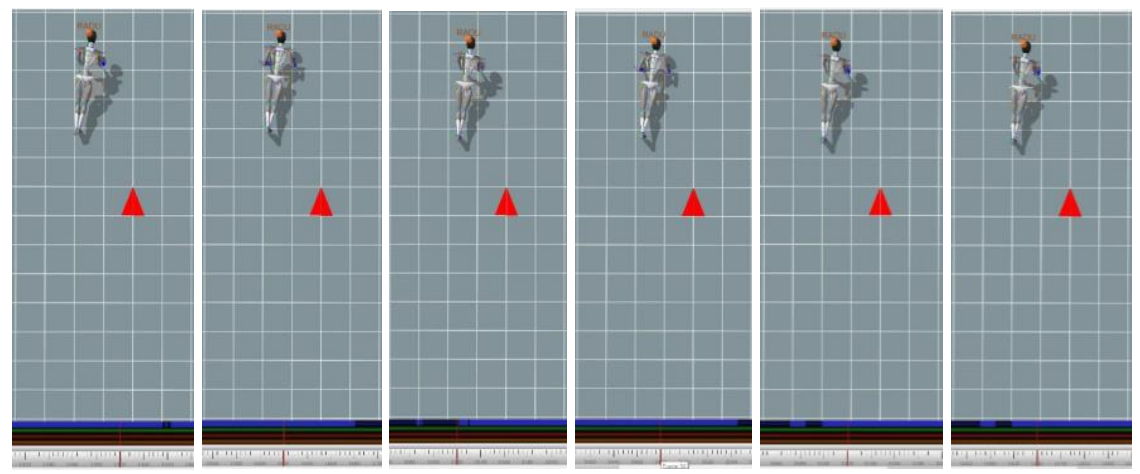

Figure 2: The most representative frames for the „Push-ups” analysis sesion, representing the transverse-cranial plane of the human subject, before the calling of "REPROCESS" function

By comparing the frames in Fig. 2, one can observe a "sliding" of the human subject towards the virtual reference marker. Also, one can notice the transfer of the contact points from the hands to the toes and vice versa, throughout the whole exercise. These two phenomena are in contradiction with the reality.
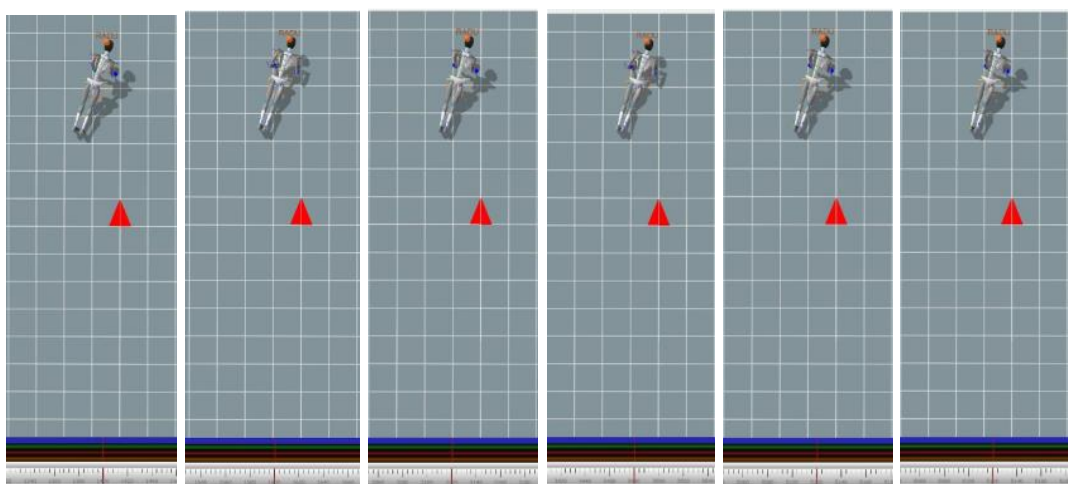

Figure 3: The most representative frames for the „Push-ups” analysis sesion, representing the transverse-cranial plane of the human subject, after the calling of "REPROCESS" function

By comparing the frames in Fig. 3, one can observe a "sliding" of the human subject's left hand towards the torso's exterior, during the flexion stages and return to the starting position, during the extension stages.

In order to make an accurate comparison between the position of the human subject, before and after the calling of "REPROCESS" function, with respect to the global coordinate system, the analysis was carried out on the basis of the charts of Fig. 10, Fig. 17, Fig. 18, Fig. 19, Fig. 20, Fig. 21, Fig. 22, Fig. 23, Fig. 24, Fig. 25 and Fig. 26.
Fig. 4, fig. 5, fig. 6, fig. 7, fig. 8 and fig. 9 are representations of the left sagittal plane, of the human subject under analysis, at various moments in time, having the arms extended or flexed (see fig. 31). The analysis based on the above-mentioned figures is coarse and it's relating to the virtual reference plane.

The exact positions of the body segments, resulting in the positions of the virtual dummy in relation to the global coordinate system before and after the calling of "REPROCESS" function, are represented in the charts of fig. 24, fig. 27, fig. 28 and fig. 31. 


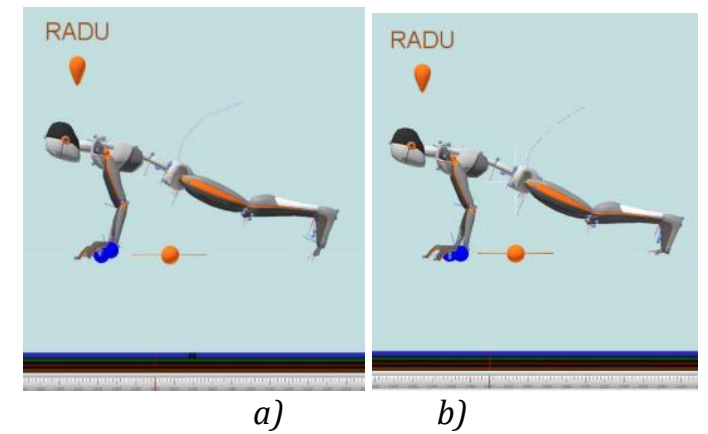

Figure 4: The first chosen frame to represent the left sagittal plane of the human subject, the latter having the arms extended, during the pushup session, before and after the calling of "REPROCESS" function

Analyzing the two views of fig. 4, in which the virtual dummy sits in the „Push-ups” position having the arms extended, one can observe the following aspects:

- in the a) view, the virtual dummy has the toes in contact with the virtual reference plane, the flexion angle, at the level of the joints between the metatarsals and the phalanges, being very small, which contradicts the reality, whereas, in the b) view, the virtual dummy has the toes in contact with the virtual reference plane, the flexion angle, at the level of the joints between the metatarsals and the phalanges, being normal for this posture, which corresponds to the real situation.

This is confirmed by the chart of the variation of the Flexion / Extension angle of the joint between the metatarsals and the phalanges, before and after calling of "REPROCESS" function (see fig. 17);
- also, although the contact between the toes and the virtual reference plane (this being a digital representation of the floor) can be visually notice, both before and after the calling of "REPROCESS" function, due to the flexion of the joints between the toes that clearly imply the existence of that contact with the floor, however, the XSens's MVN software does not represent these contact points, which means that they do not exist, only the once at the hand level being considered.

This is contrary to reality, being also proved by the comparative analysis between the chart of the variations in time of the toes position's "Z" coordinate and the chart of the variations in time of the hand position's "Z" coordinate (see fig. 25 and fig. 26);

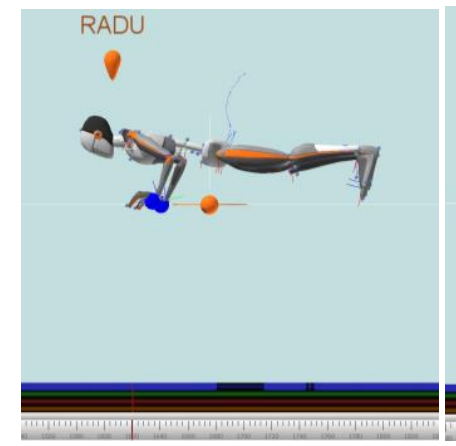

a)

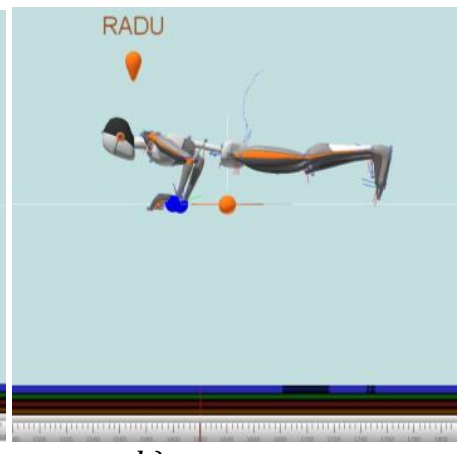

b)

Figure 5: The second chosen frame to represent the left sagittal plane of the human subject, the latter having the arms flexed, during the pushup session, before and after the calling of "REPROCESS" function

Analyzing the two views of fig. 5, in which the virtual dummy sits in the „Push-ups” position having the arms flexed, one can observe the following aspects:

- in the a) view, the virtual dummy has the toes in contact with the virtual reference plane, the flexion angle, at the level of the joints between the metatarsals and the phalanges, being very small, which doesn't contradicts the reality, whereas, in the b) view, the virtual dummy has the toes in contact with the virtual reference plane, the flexion angle, at the level of the joints between the metatarsals and the phalanges, being normal for this posture, which corresponds to the real situation.

This is confirmed by the chart of the variation of the Flexion / Extension angle of the joint between the metatarsals and the phalanges, before and after the calling of "REPROCESS" function (see fig. 17);

- also, one can notice that there is no contact between the toes and the virtual reference plan, both before and after the calling of "REPROCESS" function, therefore the XSens's MVN software does 
not represent these contact points, taking into account only the ones situated at the hand level.

This contradicts the reality, as the human subject doesn't have his feet suspended up in the air and this is also proved by the comparative analysis between the chart of the variations in time of the toes position's "Z" coordinate and the chart of the variations in time of the hand position's "Z" coordinate (see fig. 25 and fig. 26);

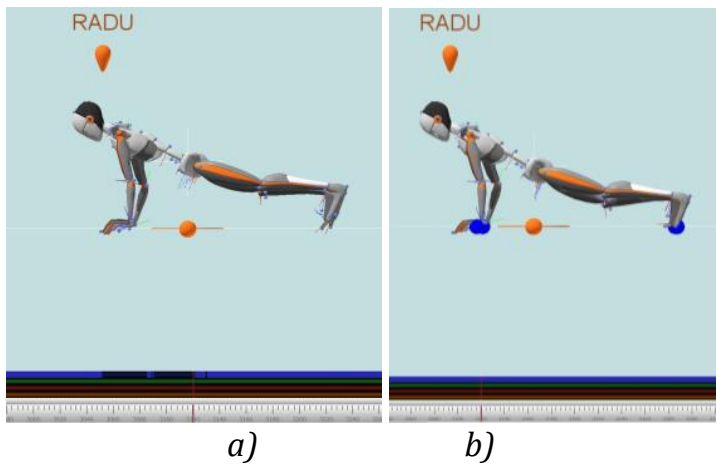

Figure 6: The third chosen frame to represent the left sagittal plane of the human subject, the latter having the arms extended, during the pushup session, before and after the calling of "REPROCESS" function

Analyzing the two views of fig.6, in which the virtual dummy sits in the „Push-ups” position having the arms extended, one can observe the following aspects:

- in the a) view, the virtual dummy has the toes in contact with the virtual reference plane, the flexion angle, at the level of the joints between the metatarsals and the phalanges, being very small, which contradicts the reality, whereas, in the b) view, the virtual dummy has the toes in contact with the virtual reference plane, the flexion angle, at the level of the joints between the metatarsals and the phalanges, being normal for this posture, which corresponds to the real situation. This is confirmed by the chart of the variation of the Flexion / Extension angle of the joint between the metatarsals and the phalanges, before and after the calling of "REPROCESS" function (see fig. 17);
- also, although the contact between the toes and the virtual reference plane (this being a digital representation of the floor) can be visually notice, both before and after the calling of "REPROCESS" function, due to the flexion of the joints between the toes that clearly imply the existence of that contact with the floor, however, the XSens's MVN software represent these contact points only after the calling of "REPROCESS" function, those being taken in consideration alongside the once situated at the hand level. Before the calling of "REPROCESS" function, the lack of contact points situated at the toes level is contrary to reality, being also proved by the comparative analysis between the chart of the variations in time of the toes position's "Z" coordinate and the chart of the variations in time of the hand position's "Z" coordinate (see fig. 25 and fig. 26);

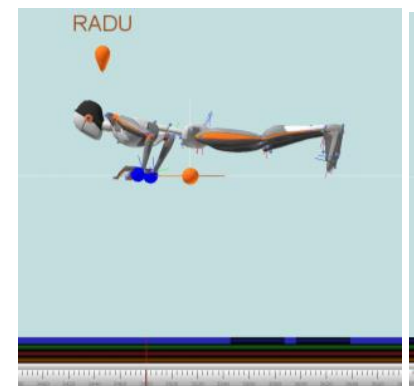

a)

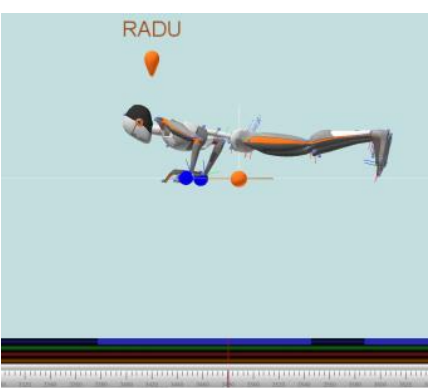

b)

Figure 7: The forth chosen frame to represent the left sagittal plane of the human subject, the latter having the arms flexed, during the pushup session, before and after the calling of "REPROCESS" function

Analyzing the two views of fig.7, in which the virtual dummy sits in the „Push-ups” position having the arms flexed, one can observe the following aspects:

- in the a) view, the virtual dummy has the toes in contact with the virtual reference plane, the flexion angle, at the level of the joints between the metatarsals and the phalanges, being very small, which doesn't contradicts the reality, whereas, in the b) view, the virtual dummy has the toes in contact with the virtual reference plane, the flexion angle, at 
the level of the joints between the metatarsals and the phalanges, being normal for this posture, which corresponds to the real situation. This is confirmed by the chart of the variation of the Flexion/Extension angle of the joint between the metatarsals and the phalanges, before and after the calling of "REPROCESS" function (see fig.17);

- also, one can notice that there is no contact between the toes and the virtual reference plan, both before and after the calling of "REPROCESS" function, therefore the XSens's MVN software does not represent these contact points, taking into account only the ones situated at the hand level. This contradicts the reality, as the human subject doesn't have his feet suspended up in the air and this is also proved by the comparative analysis between the chart of the variations in time of the toes position's "Z" coordinate and the chart of the variations in time of the hand position's "Z" coordinate (see fig.25 and fig.26);

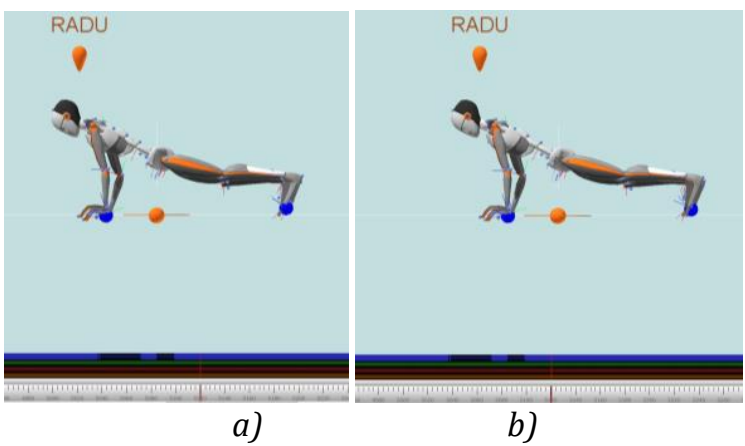

Figure 8: The fifth chosen frame to represent the left sagittal plane of the human subject, the latter having the arms extended, during the pushup session, before and after the calling of "REPROCESS" function

Analyzing the two views of fig.8, in which the virtual dummy sits in the "Push-ups" position having the arms extended, one can observe the following aspects:

- in the a) view, the virtual dummy has the toes in contact with the virtual reference plane, the flexion angle, at the level of the joints between the metatarsals and the phalanges, being very small, which contradicts the reality, whereas, in the b) view, the virtual dummy has the toes in contact with the virtual reference plane, the flexion angle, at the level of the joints between the metatarsals and the phalanges, being normal for this posture, which corresponds to the real situation. This is confirmed by the chart of the variation of the Flexion / Extension angle of the joint between the metatarsals and the phalanges, before and after the calling of "REPROCESS" function (see fig.17);

- also, although the contact between the toes and the virtual reference plane (this being a digital representation of the floor) can be visually notice, both before and after the calling of "REPROCESS" function, however, the XSens's MVN software doesn't represent all these contact points, representing only one contact point for the level of hands and one for the level of toes This is contrary to reality, being also proved by the comparative analysis between the chart of the variations in time of the toes position's "Z" coordinate and the chart of the variations in time of the hand position's "Z" coordinate (see fig.25 and fig.26);

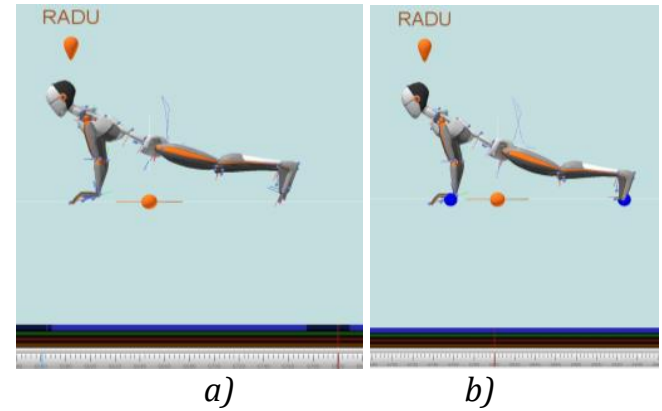

Figure 9: The sixth chosen frame to represent the left sagittal plane of the human subject, the latter having the arms extended, during the pushup session, before and after the calling of "REPROCESS" function

Analyzing the two views of fig.9, in which the virtual dummy sits in the "Push-ups” position having the arms extended, one can observe the following aspects:

- in the a) view, the virtual dummy has the toes in contact with the virtual reference plane, the flexion angle, at the level of the joints between the metatarsals and the phalanges, being very small, which contradicts the reality, whereas, in the b) view, the virtual dummy has the toes in contact with the virtual reference plane, the flexion angle, at the level of the joints between the metatarsals and the phalanges, being normal for this posture, which corresponds to the real situation. This is confirmed by the chart of the variation of the Flexion / Extension angle of the joint between the metatarsals and the phalanges, before and after the calling of "REPROCESS" function (see fig.17);

- also, although the contact between the toes and the virtual reference plane (this being a digital representation of the floor) can be visually notice, both before and after the calling of "REPROCESS" function, due to the flexion of the joints between the toes that clearly imply the existence of that contact with the floor, however, the XSens's MVN software represent these contact points only after the calling of "REPROCESS" function, those being taken in consideration alongside the once situated at the hand level. Before the calling of "REPROCESS" function, the lack of contact points situated at the toes level is contrary to reality, being also proved by the comparative analysis between the chart of the variations in time of the toes position's "Z" coordinate and the chart of the variations in time of 
the hand position's "Z" coordinate (see fig.25 and fig.26);

Fig.11, Fig.12, Fig.13, Fig.14, Fig.15 and Fig.16 are representations of the transverse-caudal plane, of the human subject under analysis, at various moments in time, having the arms extended or flexed (see Fig.10).

The analysis based on the above-mentioned figures is coarse and it's relating to the X0Z plane.

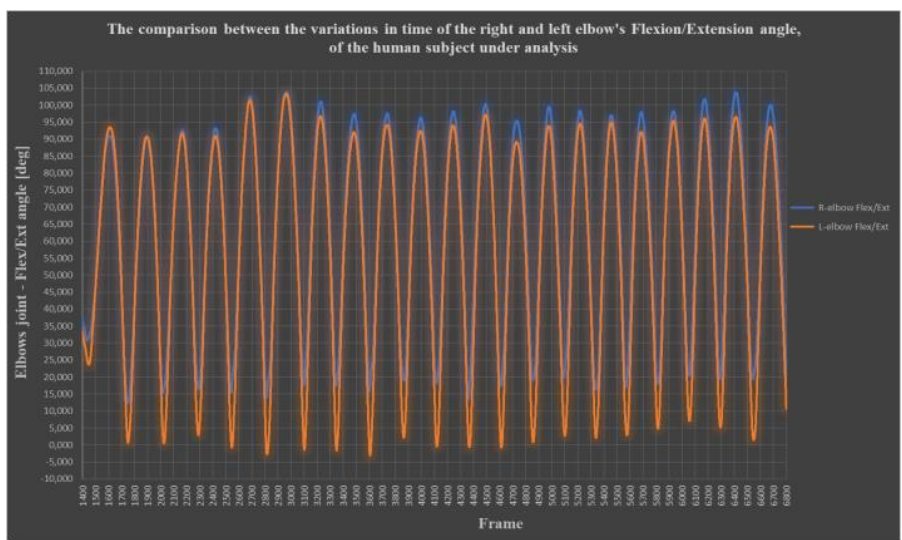

Figure 10: The comparison between the variations in time of the right and left elbow's Flexion/Extension angle, of the human subject under analysis

The exact positions of the body segments, resulting in the positions of the virtual dummy in relation to the XOZ plane, before and after the calling of "REPROCESS" function, are represented in the charts of Fig.18, Fig.19, Fig.20, Fig.21, Fig.22 and Fig.23.

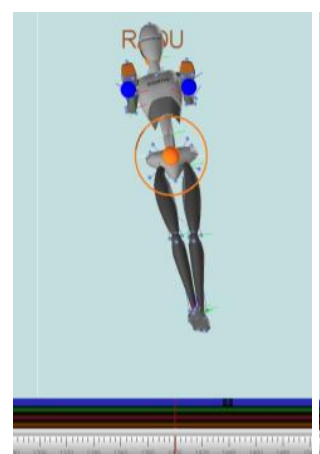

a)

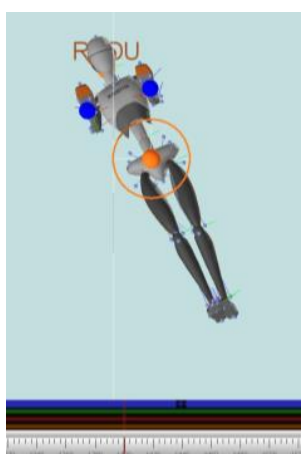

b)
Figure 11: The first chosen frame to represent the transverse-caudal plane of the human subject, the latter having the arms extended, during the „Pushups" session, before and after the calling of "REPROCESS" function

Analyzing the two views of Fig.11, in which the virtual dummy sits in the „Push-ups” position having the arms extended, one can observe the following aspects:

- in the a) view, the virtual dummy has its feet overleaped, which contradicts reality, while, in the b) view, the virtual dummy has its feet only slightly overlapped, this possibly being just a problem of estimating the width of the legs, in this case, the situation being much closer to the reality.
This is also confirmed by the chart of the variations in time of the toes position's " $\mathrm{X}$ " and " $\mathrm{Y}$ " coordinate, before and after calling the "REPROCESS" function (see Fig.19 and Fig.22 respectively);

- in both views there are two contact points with the virtual reference plan, located at the level of each palm, at the level of the toes there are no contact points, as if the human subject is having his feet suspended up in the air. Those statements are being confirmed by analyzing the chart of the variations in time of the toes position's "Z" coordinate, before and after calling the "REPROCESS" function (see Fig.25) and by the comparative analysis of the latter, with the chart of the variations in time of the hands position's "Z" coordinate (see Fig.26);

- in the b) view, the virtual dummy intersects, at its neck level, with the X0Z plane, while in the a) view, the intersection mentioned above is not visible.

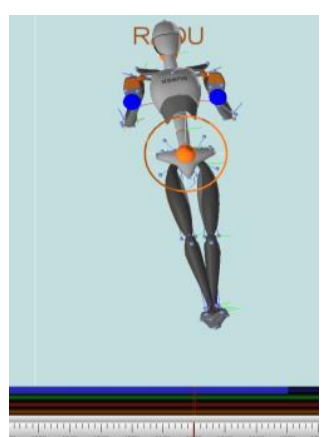

a)

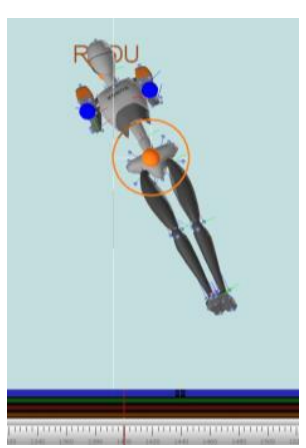

b)
Figure 12: The second chosen frame to represent the transverse-caudal plane of the human subject, the latter having the arms flexed, during the "Push-ups” session, before and after the calling of "REPROCESS" function 
Analyzing the two views of Fig.12, in which the virtual dummy sits in the „Push-ups” position having the arms flexed, one can observe the following aspects:

- in the a) view, the virtual dummy has its feet overleaped, its tibias being crossed, which contradicts reality, while, in the b) view, the virtual dummy has its feet only slightly overlapped, this possibly being just a problem of estimating the width of the legs, in this case, the situation being much closer to the reality. This is also confirmed by the chart of the variations in time of the toes position's "X" and "Y" coordinate, before and after calling the "REPROCESS" function (see Fig.19 and Fig.22 respectively);

- in both views there are two contact points with the virtual reference plan, located at the level of each palm, at the level of the toes there are no contact points, as if the human subject is having his feet suspended up in the air. Those statements are being confirmed by analyzing the chart of the variations in time of the toes position's "Z" coordinate, before and after calling the "REPROCESS" function (see Fig.25) and by the comparative analysis of the latter, with the chart of the variations in time of the hands position's "Z" coordinate (see Fig.26);

- in the b) view, the virtual dummy intersects, at its neck level, with the XOZ plane, while in the a) view, the intersection mentioned above is not visible.

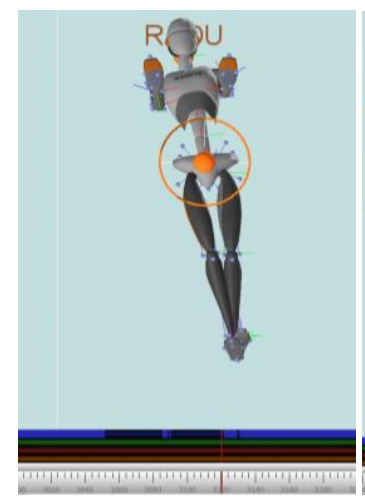

a)

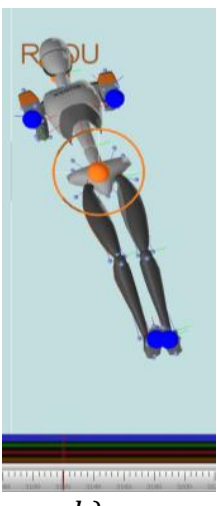

b)
Figure 13: The third chosen frame to represent the transverse-caudal plane of the human subject, the latter having the arms extended, during the „Pushups" session, before and after the calling of "REPROCESS" function

Analyzing the two views of Fig.13, in which the virtual dummy sits in the „Push-ups” position having the arms extended, one can observe the following aspects:

- in the a) view, the virtual dummy has its feet overleaped, its tibias being crossed, which contradicts reality, while, in the b) view, the virtual dummy has its feet only slightly overlapped, at the level of the toes, this possibly being just a problem of estimating the width of the legs, in this case, the situation being much closer to the reality. This is also confirmed by the chart of the variations in time of the toes position's " $\mathrm{X}$ " and "Y" coordinate, before and after calling the "REPROCESS" function (see Fig.19 and Fig.22 respectively);

- in the a) view, there are no contact points with the virtual reference plan, as if the human subject is floating in the air. Those statements are being confirmed by analyzing the chart of the variations in time of the toes position's "Z" coordinate, before and after calling the "REPROCESS" function (see Fig.25) and by the comparative analysis of the latter, with the chart of the variations in time of the hands position's "Z" coordinate (see Fig.26);

- in the b) view, the virtual dummy it's situated closer to the X0Z plane then it's in the a) view. This aspect is confirmed by the chart of the variations in time of the hands position's " $\mathrm{X}$ " and "Y" coordinate, before and after calling the "REPROCESS" function (see Fig.20 and Fig.23 respectively).

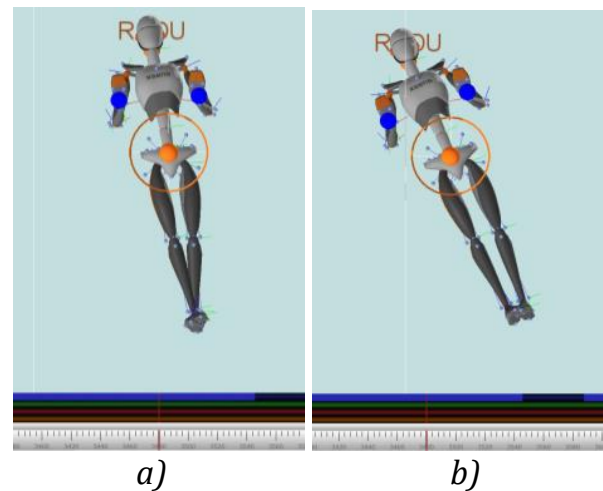

Figure 14: The forth chosen frame to represent the transverse-caudal plane of the human subject, the latter having the arms flexed, during the "Push-ups” session, before and after the calling of "REPROCESS" function

Analyzing the two views of Fig.14, in which the virtual dummy sits in the „Push-ups” position having the arms flexed, one can observe the following aspects:

- in the a) view, the virtual dummy has its feet overleaped, its tibias being crossed, which contradicts reality, while, in the b) view, the virtual dummy has its feet only slightly overlapped, at the level of the toes, this possibly being just a problem of estimating the width of the legs, in this case, the situation being much closer to the reality. This is also confirmed by the chart of the variations in time of the toes position's " $\mathrm{X}$ " and " $\mathrm{Y}$ " coordinate, before and after calling the "REPROCESS" function (see Fig.19 and Fig.22 respectively).

- in both views there are two contact points with the virtual reference plan, located at the level of each palm, at the level of the toes there are no contact points, as if the human subject is having his feet 
suspended up in the air. Those statements are being confirmed by analyzing the chart of the variations in time of the toes position's "Z" coordinate, before and after calling the "REPROCESS" function (see Fig.25) and by the comparative analysis of the latter, with the chart of the variations in time of the hands position's "Z" coordinate (see Fig.26);

- in the b) view, the virtual dummy intersects, at its head level, with the X0Z plane, while in the a) view, the intersection mentioned above is not visible.
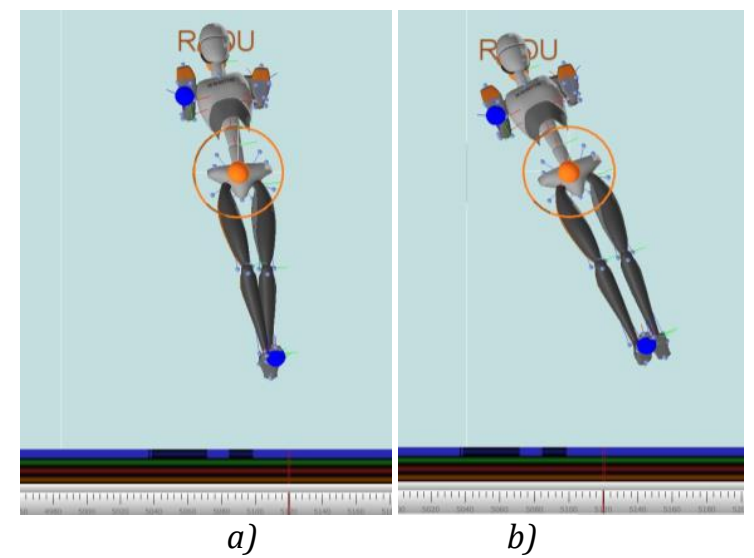

Figure 15: The fifth chosen frame to represent the transverse-caudal plane of the human subject, the latter having the arms extended, during the „Push-ups" session, before and after the calling of "REPROCESS" function

Analyzing the two views of Fig. 15, in which the virtual dummy sits in the „Push-ups” position having the arms extended, one can observe the following aspects:

- in the a) view, the virtual dummy has its feet overleaped, its tibias being crossed, which contradicts reality, while, in the b) view, the virtual dummy has its feet only slightly overlapped, at the level of the toes, this possibly being just a problem of estimating the width of the legs, in this case, the situation being much closer to the reality. This is also confirmed by the chart of the variations in time of the toes position's "X" and "Y" coordinate, before and after calling the "REPROCESS" function (see Fig.19 and Fig. 22 respectively);
- in the a) view, there are only two contact points with the virtual reference plan, situated at the level of the right arm and right toe, as if the human subject is having its left side floating in the air. Those statements are being confirmed by analyzing the chart of the variations in time of the toes position's "Z" coordinate, before and after calling the "REPROCESS" function (see Fig. 25) and by the comparative analysis of the latter, with the chart of the variations in time of the hands position's "Z" coordinate (see Fig. 26);

- in the b) view, the virtual dummy it's situated closer to the X0Z plane then it's in the a) view. This aspect is confirmed by the chart of the variations in time of the hands position's "X" and "Y" coordinate, before and after calling the "REPROCESS" function (see Fig. 19 and Fig. 22 respectively).

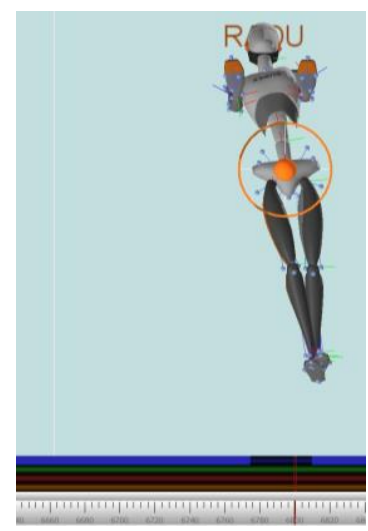

a)

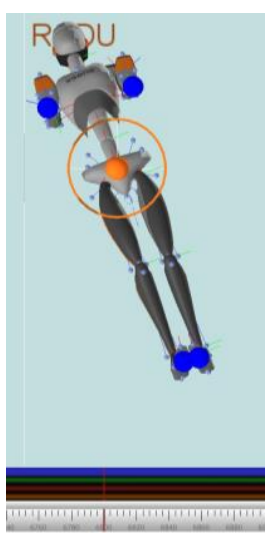

b)

Figure 16: The sixth chosen frame to represent the transverse-caudal plane of the human subject, the latter having the arms extended, during the „Push-ups" session, before and after the calling of "REPROCESS" function 
The Positioning Errors Generated by Xsens MVN Inductive System During the Analysis of „Push-ups” Exercise, using the „Single Level” Scenario, Before and After the Calling of "REPROCESS" Function

Analyzing the two views of Fig.16, in which the virtual dummy sits in the „Push-ups” position having the arms extended, one can observe the following aspects:

- in the a) view, the virtual dummy has its feet overleaped, its tibias being crossed, which contradicts reality, while, in the b) view, the virtual dummy has its feet only slightly overlapped, at the level of the toes, this possibly being just a problem of estimating the width of the legs, in this case, the situation being much closer to the reality. This is also confirmed by the chart of the variations in time of the toes position's "X" and "Y" coordinate, before and after calling the "REPROCESS" function (see Fig. 19 and Fig. 22 respectively).

- in the a) view, there are no contact points with the virtual reference plan, as if the human subject is floating in the air, while in the b) view, there are contact points both at the level of hands and toes.

Those statements are being confirmed by analyzing the chart of the variations in time of the toes position's "Z" coordinate, before and after calling the "REPROCESS" function (see Fig. 25) and by the comparative analysis of the latter, with the chart of the variations in time of the hands position's "Z" coordinate (see Fig. 26);

- in the b) view, the virtual dummy it's situated closer to the X0Z plane then it's in the a) view. This aspect is confirmed by the chart of the variations in time of the hands position's "X" and "Y" coordinate, before and after calling the "REPROCESS" function (see Fig. 20 and Fig. 23 respectively).

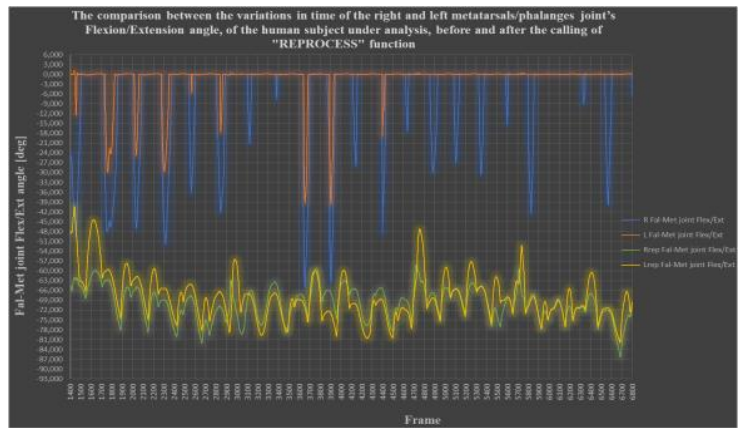

Figure 17: The comparison between the variations in time of the right and left metatarsals/phalanges joint's Flexion/Extension angle, of the human subject under analysis, before and after the calling of "REPROCESS" function

Analyzing the chart in Fig. 17, one can clearly notice the occurrence of flexion, at the level of the joints between the metatarsals and phalanges, relatively synchronous with the extension at the level of the elbow joints (see fig. 10), after the calling of "REPROCESS" function, which corresponds with the reality.

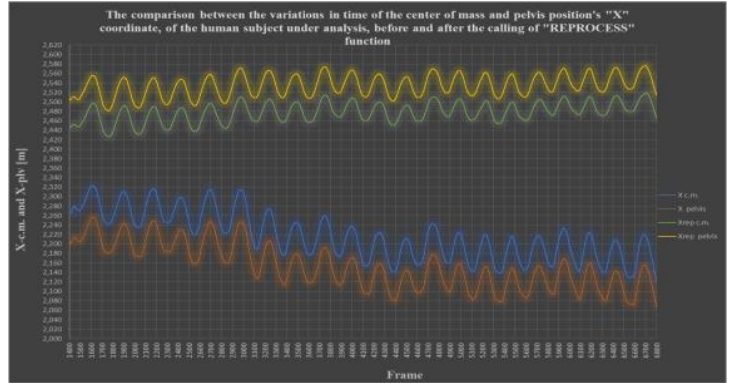

Figure 18: The comparison between the variations in time of the center of mass and pelvis position' $S$ " $X$ " coordinate, of the human subject under analysis, before and after the calling of "REPROCESS" function

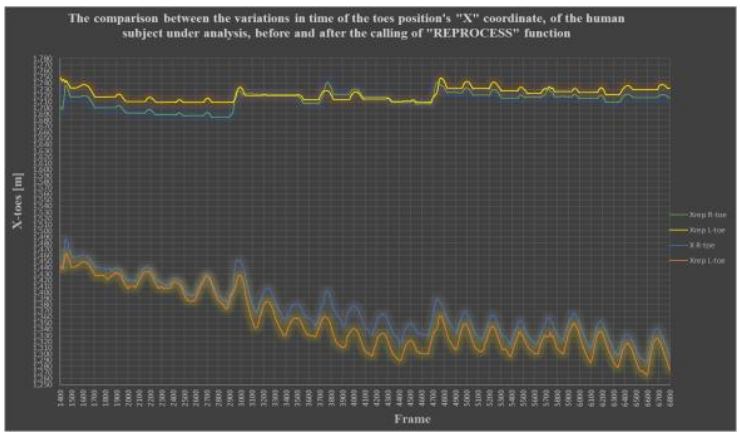

Figure 19: The comparison between the variations in time of the toes position's " $X$ " coordinate, of the human subject under analysis, before and after the calling of "REPROCESS" function

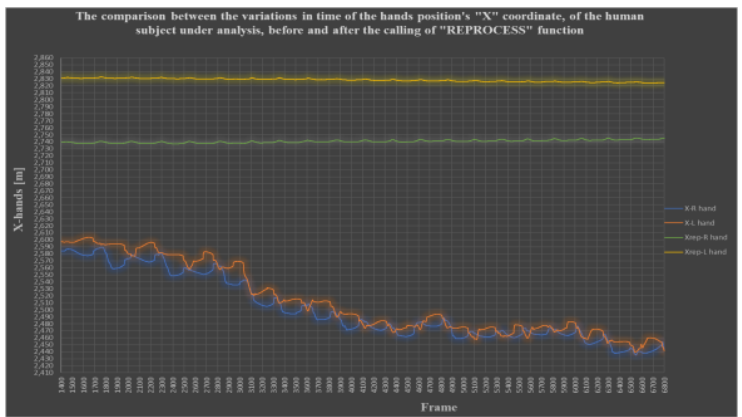

Figure 20: The comparison between the variations in time of the hands position's " $X$ " coordinate, of the human subject under analysis, before and after the calling of "REPROCESS" function

Analyzing the chart of the comparison between the variations in time of the center of mass and pelvis position's "X" coordinate, of the human subject under analysis, before and after the calling of "REPROCESS" function, in Fig. 18, the chart of the comparison between the variations in time of the toes position's " $\mathrm{X}$ " coordinate, of the human subject under analysis, before and after the calling of "REPROCESS" function, in Fig. 19 and the chart of the comparison between the variations in time of the hands position's " $\mathrm{X}$ " coordinate, of the human subject under analysis, before and after the calling of "REPROCESS" function, in Fig. 20, there can be seen a "sliding" towards the lower part of the chart, at the 
The Positioning Errors Generated by Xsens MVN Inductive System During the Analysis of „Push-ups” Exercise, using the „Single Level” Scenario, Before and After the Calling of "REPROCESS" Function

order of tens of $\mathrm{cm}$, after the calling of "REPROCESS" function, this "sliding" is reduced to several millimeters and it's manifested toward the top of the chart.

The amplitude of the signal before the calling of "REPROCESS" function is greater than the one after the calling of previously mentioned function. Moreover, from the chart in Fig. 19, it results that the signal type before the calling of "REPROCESS" function differs from the one after that.

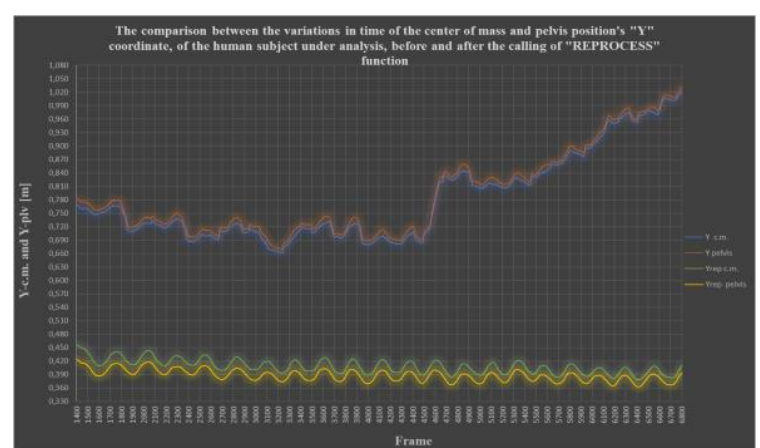

Figure 21: The comparison between the variations in time of the center of mass and pelvis position's " $Y$ " coordinate, of the human subject under analysis, before and after the calling of "REPROCESS" function

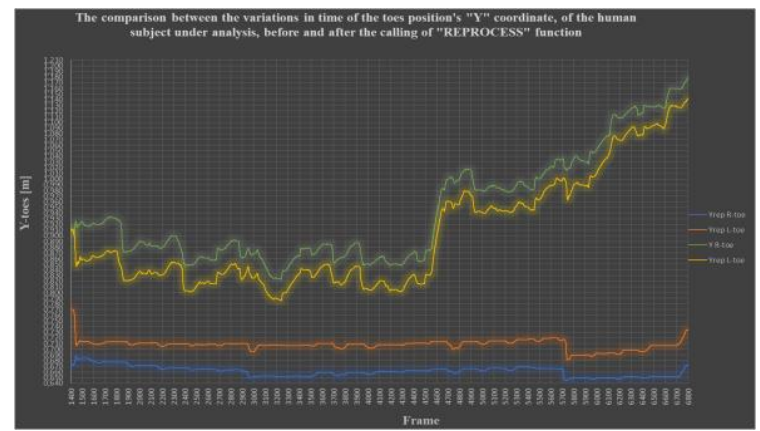

Figure 22: The comparison between the variations in time of the toes position's " $Y$ " coordinate, of the human subject under analysis, before and after the calling of "REPROCESS" function

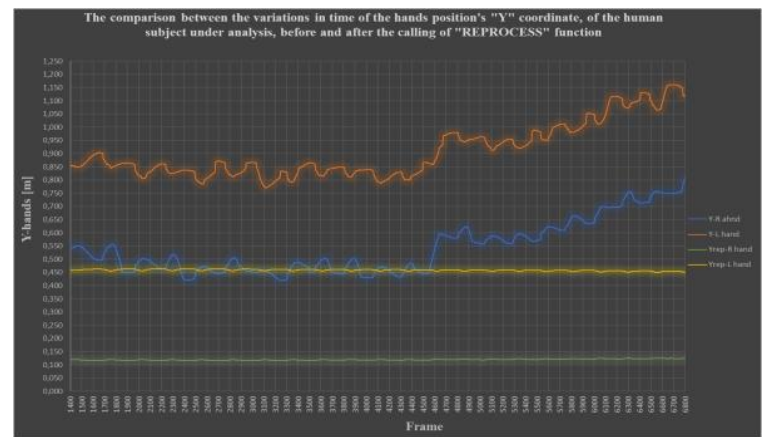

Figure 23: The comparison between the variations in time of the hands position's " $Y$ " coordinate, of the human subject under analysis, before and after the calling of "REPROCESS" function
Analyzing the chart of the comparison between the variations in time of the center of mass and pelvis position's "Y" coordinate, of the human subject under analysis, before and after the calling of "REPROCESS" function, in Fig. 21, the chart of the comparison between the variations in time of the toes position's "Y" coordinate, of the human subject under analysis, before and after the calling of "REPROCESS" function, in Fig. 22 and the chart of the comparison between the variations in time of the hands position's "Y" coordinate, of the human subject under analysis, before and after the calling of "REPROCESS" function, in Fig. 23, one can see a "sliding" at the order of tens of centimeters towards the top of the chart, after the calling of "REPROCESS" function, this "sliding" is reduced to a few mm order and it's manifested towards the bottom of the chart. The amplitude of the signal before the calling of "REPROCESS" function is greater than the one after the calling of the previously mentioned function. Also, from the analysis of the chart in Fig. 22, it can be noticed the diminishing of the overlapping of the feet after the calling of "REPROCESS" function.

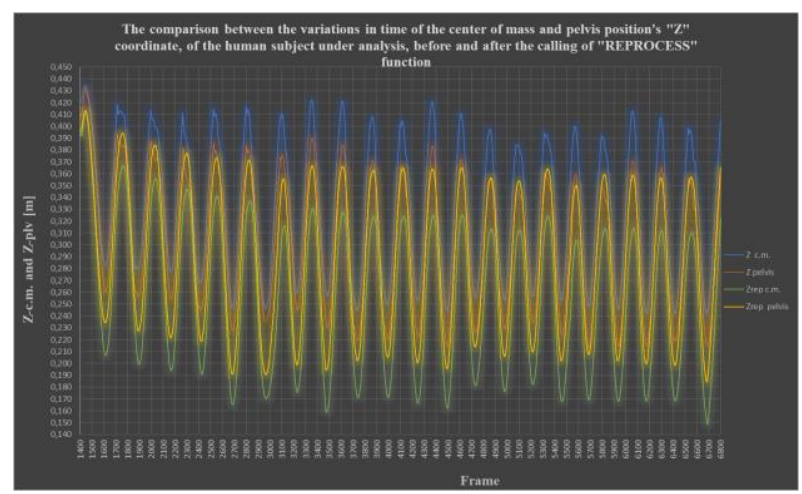

Figure 24: The comparison between the variations in time of the center of mass and pelvis position's " $Z$ " coordinate, of the human subject under analysis, before and after the calling of "REPROCESS" function

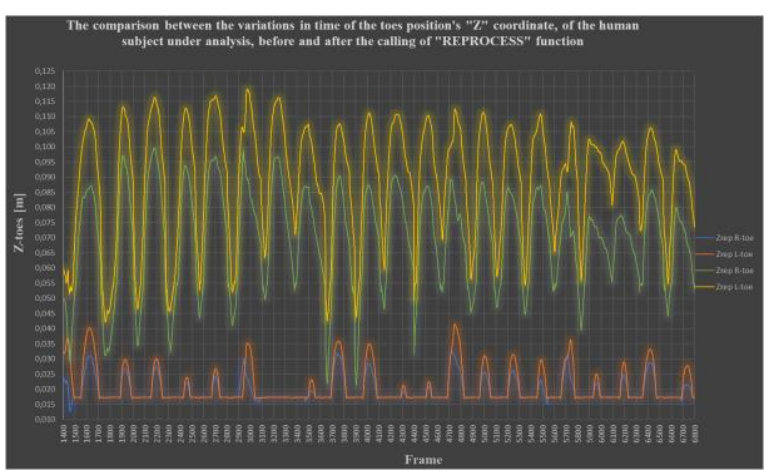

Figure 25: The comparison between the variations in time of the toes position's " $Z$ " coordinate, of the human subject under analysis, before and after the calling of "REPROCESS" function 


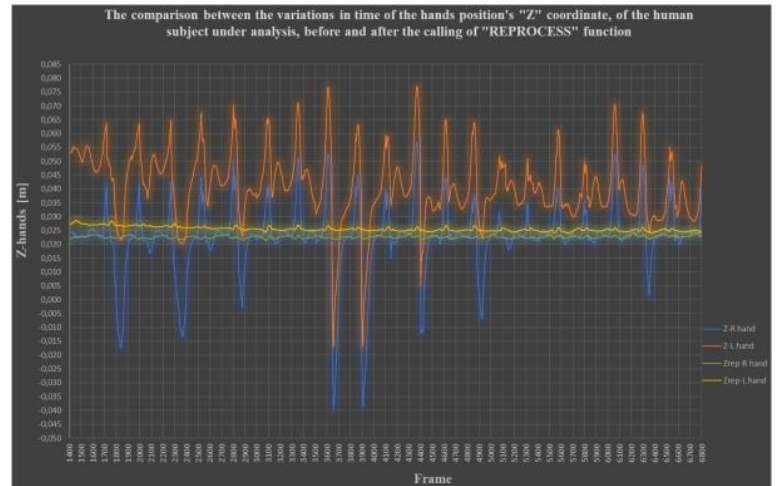

Figure 26: The comparison between the variations in time of the hands position's "Z" coordinate, of the human subject under analysis, before and after the calling of "REPROCESS" function

Analyzing the chart of the comparison between the variations in time of the center of mass and pelvis position's "Z" coordinate, of the human subject under analysis, before and after the calling of "REPROCESS" function, in Fig. 24, the chart of the comparison between the variations in time of the toes position's "Z" coordinate, of the human subject under analysis, before and after the calling of "REPROCESS" function, in Fig. 25 and the chart of the comparison between the variations in time of the hands position's "Z" coordinate, of the human subject under analysis, before and after the calling of "REPROCESS" function, in Fig. 26, one can observe that the amplitude of the signal before the calling of "REPROCESS" function is greater than the one after the calling of the above-mentioned function.
Moreover, from the analysis of the graph in Fig. 25, it results that the toes are closer to the ground after the calling of "REPROCESS" function than before that and from the analysis of the graph in Fig. 26 it results that the hands, of the human subject under analysis, are closer to the ground after the calling of "REPROCESS" function than before that and the hands positions are no longer found in the negative side of the chart.

\section{Conclusions}

a) The system in question presents both global positioning errors and local errors generated by the MEMS located on the feet.;

b) One can notice the transfer of the contact points from the palms to the toes and vice versa, throughout the entire session.

c) After the calling of "REPROCESS" function, the errors decrease but they are not completely eliminated, being reduced from the $\mathrm{cm}$ to the $\mathrm{mm}$ order.

\section{References}

[1] https://www.xsens.com/

[2] https://www.xsens.com/tags/inertial-sensors/

[3] https://www.quora.com/How-does-a-MEMSgyroscope-work

[4] https://www.gearssports.com/optical-vsinertial-motion-capture/

[5] https://www.sensorsmag.com/components/ove overv-mems-inertial-sensing-technology

[6] https://teslasuit.io/blog/motioncapture/motion-capture-what-it-is

[7] https://en.wikipedia.org/wiki/Motion_capture 